\title{
IMPACTSCHÄDEN SCHNELL UND EINFACH FINDEN
}

\author{
M. Sc. J. Rittmann 1 , Dr.-Ing. M. Rahammer', Dr.-Ing. N. Holtmann², und Prof. Dr. rer. nat. habil. M. \\ Kreutzbruck ${ }^{1}$ \\ ${ }^{1}$ IKT, Institut für Kunststofftechnik der Universität Stuttgart, Pfaffenwaldring 32, 70569 Stuttgart \\ ${ }^{2}$ edevis $\mathrm{GmbH}$, Handwerkstraße 55, 70565 Stuttgart \\ Tel.: +49 711 685-62887, E-Mail: johannes.rittmann@ikt.uni-stuttgart.de
}

\section{Zusammenfassung}

Impactschäden in Faserkunststoffverbunden wie CFK und GFK stellen hohe Herausforderungen an die zerstörungsfreie Prüftechnik (ZfP). Die anisotrope Werkstoffstruktur erschwert die Ergebnisinterpretation herkömmlicher Prüfverfahren erheblich. Die Resonante Frequenzsweep Thermografie (RFST) basiert auf dem Prinzip der lokalen Defektresonanz in Verbindung mit der bekannten Ultraschall-angeregten Thermografie. Mit ihr ist es möglich, in kürzester Zeit auf einfache Art und Weise die Existenz von signifikanten Impactschäden festzustellen. RFST nutzt eine Frequenzsweep-Anregung im unteren und mittleren Kilohertzbereich, um Defekt-, Anregungs- und Bauteilresonanzen zu aktivieren. Resonanzen verstärken die akustische Aktivierung der Defekte um ein Vielfaches und führen zu einer signifikanten Steigerung des thermischen Signals. Dieses basiert auf Rissuferreibung sowie visko-elastischer Erwärmung und wird an der Oberfläche von einer Thermografiekamera detektiert. Die Defekterkennungsschwelle hängt dabei von der Anregungsleistung sowie dem Abstand der Defekte zur Schallquelle ab. Zu diesem Zweck wurde ein Prototyp in Form eines Dreibeins mit integrierter IR-Kamera und Ultraschallanregung entwickelt. Dieser zeichnet sich durch einfache Handhabung und flexible Einsatzmöglichkeiten aus. Mithilfe von Augmented Reality wird dem Prüfer die Ergebnisinterpretation und Defektmarkierung erleichtert, indem das ausgewertete Prüfergebnis ortsgetreu auf das Bauteil projiziert wird. Vorgestellt werden erste Ergebnisse an einer Serie von Impactschäden in CFK mit variierenden Einschlagenergien und Delaminationsflächen. In diesen Bauteilen lassen sich für eine Prüfdauer von einer Minute Impactschäden mit projizierter Schadensfläche von mindestens ein Zoll Durchmesser sicher detektieren.

Keywords: Impactschäden, Faserkunststoffverbunde, Ultraschall Thermografie, Resonanz

\section{Einleitung}

Impactschäden sind kurzzeitige, punktförmige Belastungen senkrecht zur Bauteilebene. In dieser Lastrichtung sind Faserkunststoffverbunden (FKV) am anfälligsten, da dies keine primäre Faserorientierung ist. Oft genannte Ursachen sind fallengelassene Werkzeug („Tool Drop“), Hagel oder Vogelschlag („Bird Strike“). Eines der häufigsten Beispiele aus der Luftfahrt ist jedoch ein Zusammenstoß mit anderen Fahrzeugen während der Abfertigung („Ramp Crash") [1]. Je nach Energie des Einschlags/Aufpralls kommt es zu Matrixbrüchen, Delamination oder gar Faserbrüchen und einem Durchdringen des Impaktors. Bereits relativ geringe Energien erzeugen Delaminationen, obwohl rein äußerlich kein Schaden entstanden zu sein scheint („Barely Visible Impact Damage“, BVID). Das Resultat der Spannungsverteilung bei Impactbelastung ist eine mit der Tiefe anwachsende Schadensfläche. Ein solches
Schadensbild ist in Abb. 1 aus [2] abgebildet. Ähnlich einer Drei-Punkt-Biegung sind bei einem Impact Druck- bzw. Zugspannungen an den beiden Oberflächen maximal, während in der Mitte Schubspannungen maximal werden. Die Druckfestigkeit des Verbunds ist vergleichsweise hoch, weshalb es hier kaum zu Schäden kommt. Die Schubspannungen führen jedoch zu Matrixbrüchen (i), welche sich an Grenzflächen zu Delaminationen (ii) akkumulieren. Dieser Effekt wird nach unten hin durch die zunehmenden Zugspannungen zusätzlich begünstigt, weshalb das Schadenausmaß zunimmt. Aufgrund der Rotationssymmetrie der Last treten in den tiefsten Lagen noch zusätzlich Matrixrisse (iii) auf, welche parallel zur Faser verlaufen. 
Impact

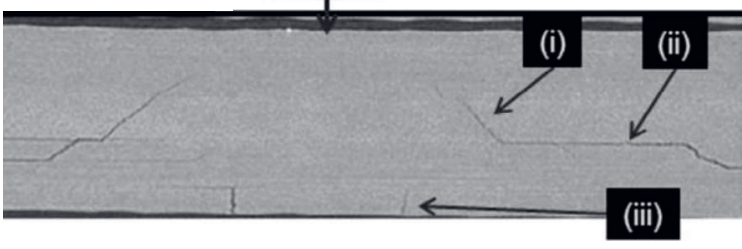

Abb. 1: Computertomografie-Schnittbild eines Impactschadens in CFK [2]

Von den aktuell bekannten Prüfverfahren eignen sich sowohl Ultraschallprüfung [3] als auch Computerthomografie [4] sehr gut für die Detektion von Impactschäden, aber nur bedingt für einen schnellen und einfachen mobilen Einsatz. Die normativ verankerten Verfahren sind entweder sehr aufwendig durchzuführen oder sehr kompliziert zu bewerten. Neben diesen beiden Standardverfahren etabliert sich die ZfP mittels Thermografie immer weiter. Hauptanwendungsgebiet der optisch angeregten Thermografie (OTT) für FKV ist die schnelle ZfP an großen, dünnen Flächen, wie sie z. B. in vielen Luftfahrtanwendungen oder bei Windkraftanlagen vorkommen. Defekte, die sich dabei sehr gut finden lassen, sind Delaminationen (z. B. aufgrund von Impact) [5], welche das Abfließen von Wärme verhindern.

Wenig bekannt ist hingegen die Ultraschallangeregte Thermografie (UTT), welche im Gegensatz zu den meisten ZfP-Verfahren ein defekt-selektives Verfahren ist. Dies bedeutet, dass Defekte ein sehr viel stärkeres Signal erzeugen, als intakte Strukturen. Diese Selektivität hat mehrere Gründe. Erstens tritt Rissuferreibung überhaupt nur an Rissen oder rissähnlichen Defekten auf. Zweitens findet man Spannungsspitzen vornehmlich in der Nähe von geschädigten Bereichen, und drittens ist die Biegesteifigkeit durch einen Defekt lokal geschwächt und die Schwingung damit erhöht. Seit Mignogna et al. [6] ist es üblich, Ultraschallschweißsonotroden aus der Kunststoffschweißtechnik für die Anregung zu verwenden. Schweißsonotroden arbeiten bei einer fixen Resonanzfrequenz (meistens 20 oder $40 \mathrm{kHz}$ ), um im Anpresspunkt so viel Energie wie möglich einzukoppeln. Sehr viel weniger Energie ist jedoch notwendig, wenn man die Resonanzfrequenz des Bauteils [7] oder des Defektes [8, 9] zur Anregung nutzt. Auf Basis dieser Entdeckungen entstand am IKT ein komplett neuer Prüfansatz basierend auf der Ultraschall-angeregten Thermografie [10], welcher bereits in einen ersten Prototypen überführt werden konnte.

\section{Das RFST-Prüfsystem}

Kernbausteine des Verfahrens sind die Anregungsquelle und die Thermografiekamera. Zur Anregung werden drei eigens für dieses Verfahren entwickelte Vakuum-Piezo-Shaker verwendet, welche sowohl mit Überdruck als auch Unterdruck betrieben werden können. Das erzeugte Vakuum dient dabei einerseits der Fixierung des Systems auf der zu prüfenden Struktur, als auch der Erzeugung eines Anpressdrucks für die Ankopplung der Shaker. Versorgt werden diese von einem Verstärker, ebenfalls isi-sys $\mathrm{GmbH}$, mit einer Wechselspannung von $60 \mathrm{~V}$ Amplitude. Das System aus Verstärker und Shakern hat seine Eigenresonanz bzw. seine höchste Effizienz im Bereich von 15-25 kHz. Aus Gründen des Lärmschutzes wird ein Bereich von $18-25 \mathrm{kHz}$ gewählt.

Aus Kosten- und Gewichtsgründen fällt die Wahl der Thermografiekamera auf ein Mikrobolometersystem. Solche Systeme sind am Markt bereits für unter $15 \mathrm{k} €$ erhältlich und liefern NETD von bis zu kleiner $30 \mathrm{mK}$ sowie eine Bildauflösung von 640 x 512 Pixel. Im vorgestellten Prototypen (Abb. 2) wird eine FLIR A655sc eingesetzt, die zudem Thermogramme mit einer Wiederholungsrate von $50 \mathrm{~Hz}$ aufnehmen kann.

Weiterhin verfügt das System über einen integrierten Miniaturprojektor. Dieser dient dazu, das automatisch ausgewertete Prüfergebnis auf die Struktur zurück zu projizieren. Diese Augmented Reality Anwendung ermöglicht dem Prüfer eine schnelle und einfache Interpretation der Ergebnisse und erlaubt es den Defektbereich exakt zu kennzeichnen. Hierzu sind Kamerasichtfeld und Projektionsfläche optisch aufeinander abgestimmt.

\section{Einfluss der Anregungsfrequenz}

Die Defektresonanzfrequenzen von Impactinduzierten Schäden liegen oftmals über 100 $\mathrm{kHz}$. Diese Frequenzen sind mit üblichen Verstärkern und Piezo-Shakern nicht effektiv erzeugbar. So konnten für die hier verwendeten Impactschäden $z$. B. LDRFrequenzen bei $114 \mathrm{kHz}$ und. $128 \mathrm{kHz}$ identifiziert werden.

Wie bereits erwähnt lassen sich aber noch weitere optimale Frequenzen nutzen. Die elektrische Anregung z. B. hat ihr Optimum im Bereich um die $20,5 \mathrm{kHz}$, wie die blaue Messkurve in Abb. 3 zeigt. Für sehr viel höhere Frequenzen ist der genutzte Verstärker nicht ausgelegt. Der Piezo-Shaker selbst hat ebenfalls natürliche Eigenresonanzen. Bei diesen Frequenzen kann er die elektrische Energie besonders effizient in mechanische 
Schwingung umsetzen. Diese sind 21,8, 23,7 und $25,4 \mathrm{kHz}$ und ebenfalls in Abb. 3 dargestellt. Ein Vergleich der beiden Spektren zeigt, dass das kombinierte Optimum im Bereich 20-22 kHz liegt.

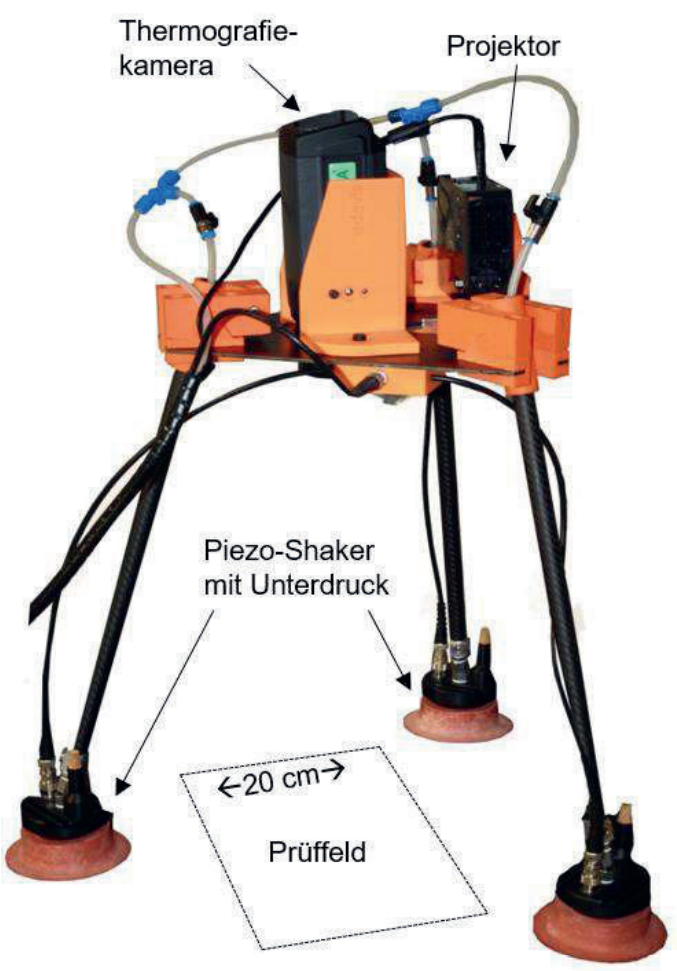

Abb. 2: Prototyp für ein mobiles RFST System

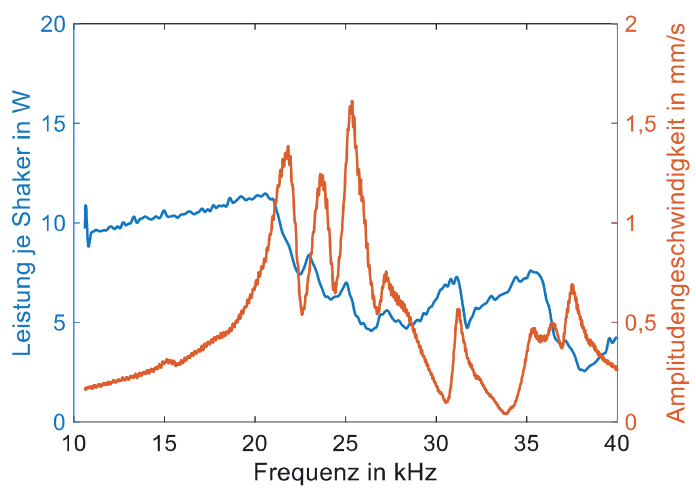

Abb. 3: Elektrische Leistung des Verstärkers (blau) und Amplitudengeschwindigkeit eines Shakers (rot) in Abhängigkeit der Frequenz

Dieser Umstand ist auch in den folgenden beiden Prüfergebnissen (Abb. 4) dargestellt. Diese zeigen jeweils ein nach der RFSTMethode ausgewertetes Amplitudenbild von einer Platte mit vier Impactschäden im Sichtbereich der Kamera. Schon eine subjektive Betrachtung zeigt, dass das schmalere, optimierte Frequenzband eine effektivere Anregung der Defekte und damit eine bessere Detektierbarkeit bewirkt.
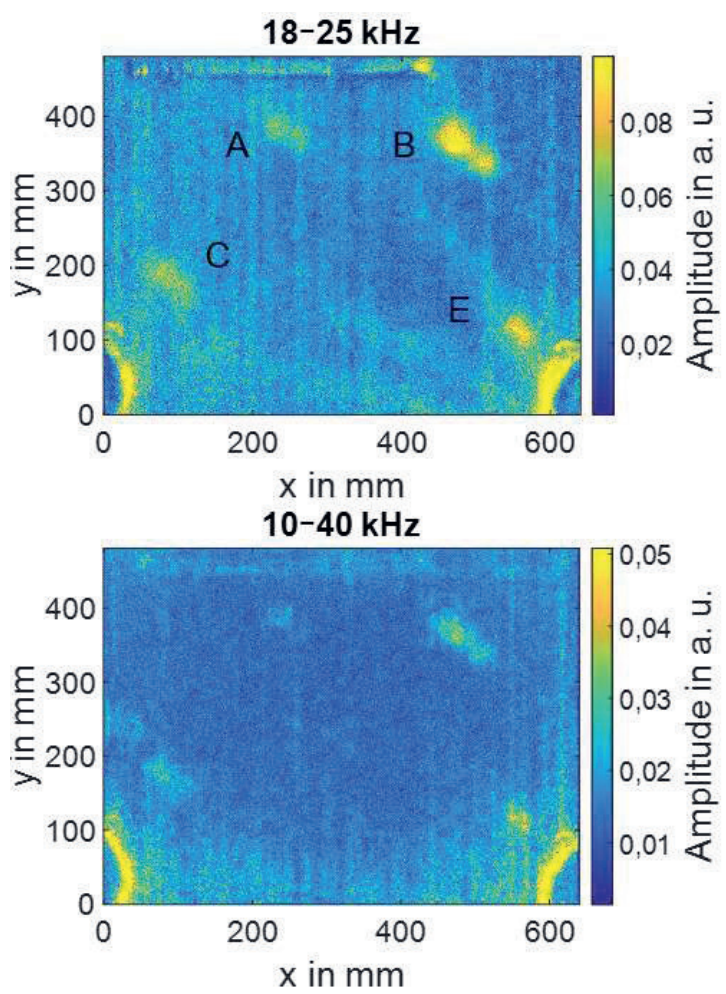

Abb. 4: Prüfergebnisse für verschiedene Anregungsbandbreiten

\section{Einfluss der Anregungsleistung}

Die Defekterkennungsrate skaliert direkt mit der Wärme, welche am Defekt umgesetzt wird. Diese erhöht sich mit steigender Ultraschallamplitude. Es wurde daher schon immer versucht, die elektrische Leistung am Piezo-Shaker zu erhöhen. Eine Testreihe mit variabler Anregungsleistung ist in Abb. 5 . dargestellt und verdeutlicht diesen Zusammenhang.

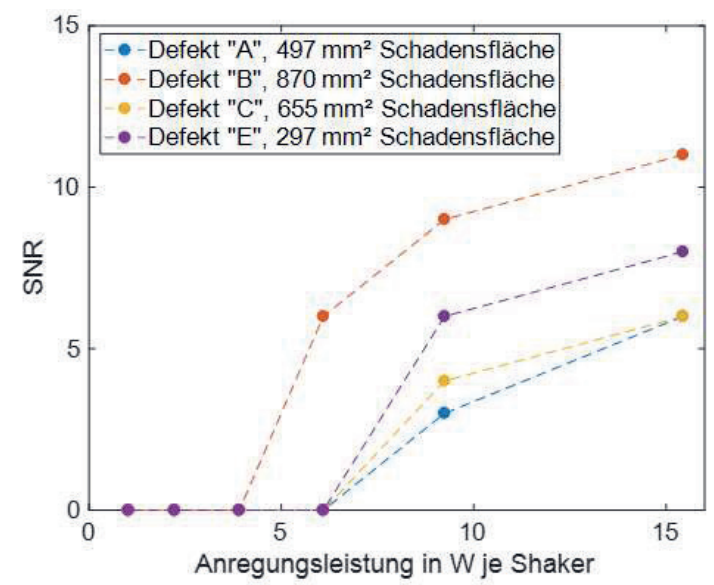

Abb. 5: Defekterkennung in Abhängigkeit der Anregungsleistung 


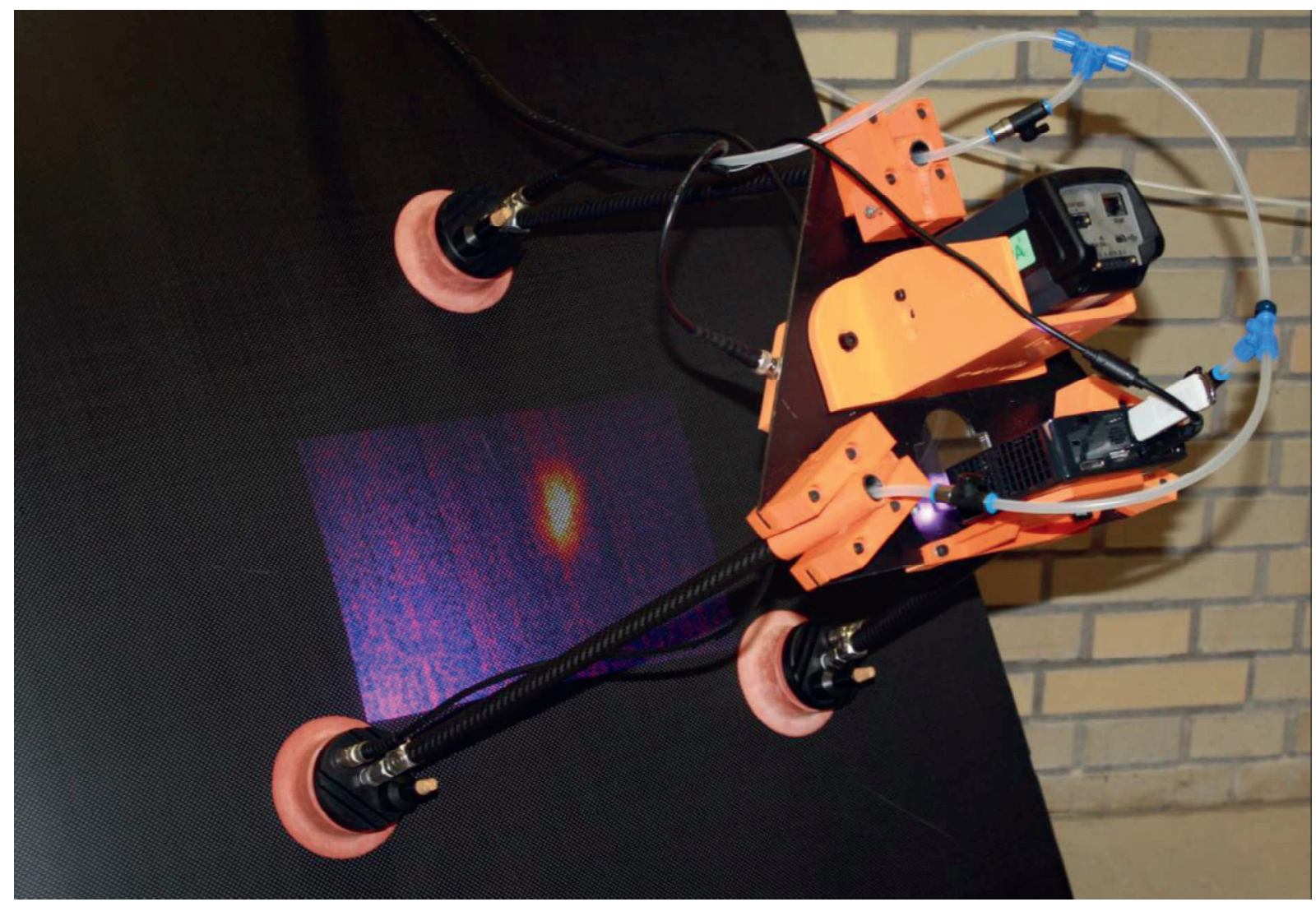

\section{Abb. 6: Mobiles RFST-Prüfsystem im Einsatz}

$\mathrm{Ab}$ einer Leistung von $8 \mathrm{~W}$ je Shaker lassen sich alle vier Defekte detektieren. Anzumerken ist hierbei, dass die Defektgröße nur teilweise Einfluss auf die Erkennungsrate hat. Ein hohes SNR kann auch für vergleichsweise kleine Defekte auftreten, wenn diese sich sehr nah an der Anregungsquelle befinden (vgl. Defekt „E“ in Abb. 5).

\section{Gesamtergebnis}

In Abb. 6 ist das Prüfsystem im Einsatz abgebildet, welches eine ca. DIN A4 große Fläche prüfen kann. Mit wahlweise Überdruck (Venturi-Prinzip) oder Unterdruck lässt es sich am zu prüfenden Bauteil fixieren und leitet dabei gleichzeitig über die Shaker in den Saugfüßen den Schall ein. Die durchströmende Luft dient zusätzlich der aktiven Kühlung der Piezo-Shaker, deren Langlebigkeit so verbessert wird. Parallel zur Beschallung nimmt die Thermografiekamera eine Thermogrammsequenz auf, welche unmittelbar online mittels Fast Fourier Transformation verarbeitet wird, um sofort ein Prüfergebnis zu erhalten. Dieses wird anschließend ortsgetreu auf die Bauteiloberfläche projiziert und ermöglicht dem Prüfer so eine schnelle und einfache Interpretation der Ergebnisse. Im Beispiel Abb. 6 ist z. B. ein Defekt vorhanden und wird in einer eindeutigen Falschfarbendarstellung abgebildet.

Insgesamt acht ähnliche Bauteile, zwei geviertelte, $3 \mathrm{~mm}$ dicke CFK-Dächer eines Automobils, wurden mit je 5 Impactschäden versehen. Beispielhaft sind die Front- und Rückseite eines Viertel-Daches in Abb. 7 dargestellt. Die Impactenergien und daraus resultierenden Schadensgrößen reichen von $4,5 \mathrm{~J}$ und $15 \mathrm{~mm}^{2}$ bis 22,4 $\mathrm{J}$ und $1704 \mathrm{~mm}^{2}$. 

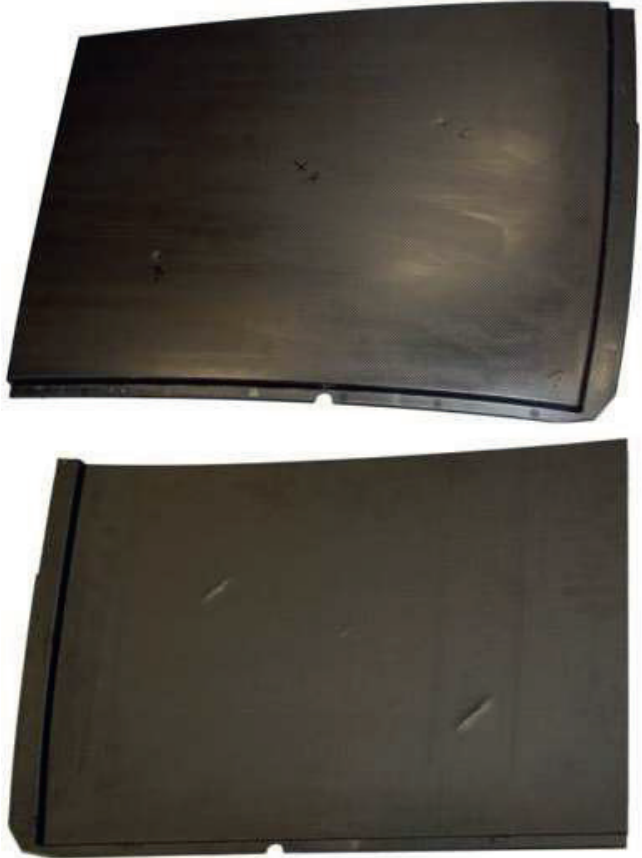

Abb.7: Viertel-Dach aus CFK mit Impactschäden. Oben: Frontseite mit Markierungen. Unten: Rückseite mit prominenten Delaminationen.

Die Prüfung dieser Bauteile für zu den in Abb. 8 dargestellten Ergebnissen. Auch hier ist die Detektionsqualität mit dem SNR-Wert angegeben, welcher aus den jeweiligen Amplitudenbildern (siehe [10]) extrahiert wurde.

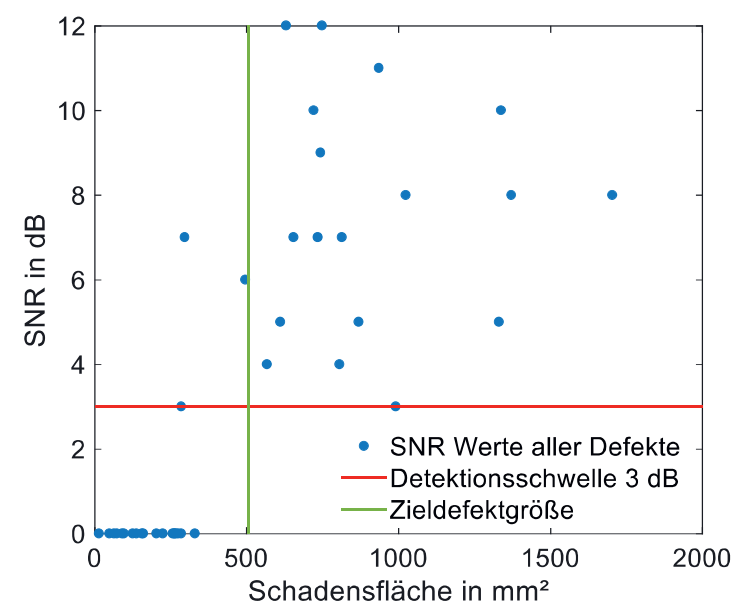

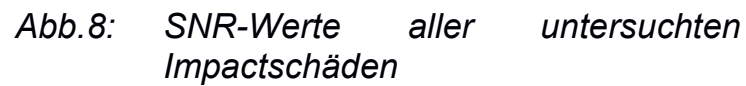

Die Detektionsschwelle, ab der ein Defekt als erkannt deklariert wird (hier: $3 \mathrm{~dB}$ ), muss für jedes Prüfverfahren und Prüfsystem neu definiert werden. Sie muss ebenso für jeden Anwendungsfall neu identifiziert werden und hängt neben dem Bauteil und Werkstoff auch von der Art der Auswertung (automatisch, manuell) ab. Für die hier durchgeführte, manuelle Bewertung der Prüfergebnisse ist ein Schwellwert von $3 \mathrm{~dB}$ gerechtfertigt, wie Abb. 9 zeigt. Der Defekt oben rechts im Bild ist mit einem SNR von $3 \mathrm{~dB}$ für das menschliche Auge noch eindeutig erkennbar. Ein zweiter Defekt, der ebenfalls im Prüfbereich liegt, ist hingegen nicht mehr erkennbar.

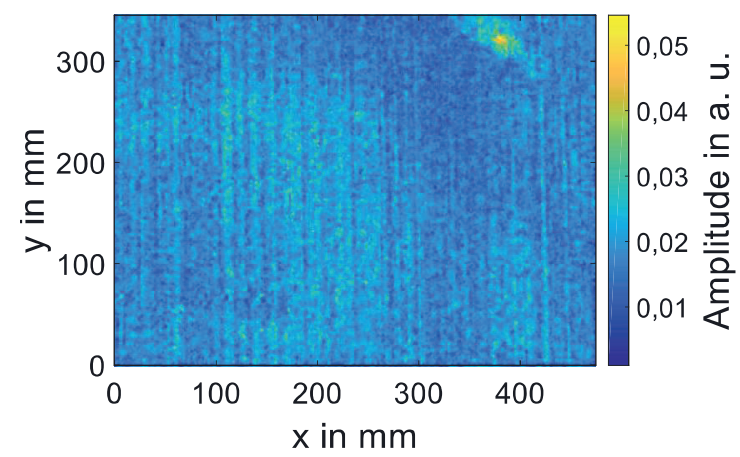

Abb. 9: Prüfergebnis einer CFK-Platte mit zwei Impactschäden ( $0 \mathrm{~dB}$ und $3 \mathrm{~dB}$ )

Wie Abb. 8 zeigt, lassen sich für die hier verwendeten Bauteile mit Impactschäden alle Defekte deren projizierte Schadensfläche größer als ein Quadratzoll beträgt eindeutig detektieren. Aus Zeit- und Kostengründen wurde auf eine ausführliche POD-Studie (Probability of Detection) verzichtet, die Reproduzierbarkeit des Verfahrens wurde aber bereits in früheren Veröffentlichungen nachgewiesen [11].

\section{Fazit}

Im Rahmen eines gemeinsamen Projektes mit der edevis $\mathrm{GmbH}$, gefördert durch das BMWi im Rahmen des Zentralen Innovationsprogramm Mittelstand, konnte ein völlig neues zerstörungsfreies Prüfverfahren entwickelt werden. Dies wurde gezielt für den Zweck der schnellen und einfach interpretierbaren Prüfung auf Impactschäden entworfen. Es erlaubt eine mobile Prüfung von Impact-gefährdeten FKV-Bauteilen mit einer Prüfdauer von einer Minute. Das Ergebnis wird ortsgetreu auf die Oberfläche zurückprojiziert und erlaubt es so dem Prüfer Defekte direkt zuzuordnen. Aufgrund der inhärenten Dunkelfeldmethode sind die Prüfergebnisse auch von ungeschultem Personal leicht zu interpretieren, was die Flexibilität des Systems nochmals erhöht.

Anhand eines exemplarischen CFK-Bauteils konnte gezeigt werden, dass relevante Schäden sicher detektiert werden können. Grundlagenuntersuchungen deuten weiterhin an, dass die Effizienz des Verfahrens bezogen auf Prüfzeit und Detektionsrate durch eine 
Optimierung der Anregungshardware noch deutlich gesteigert werden können.

\section{Literaturnachweis}

[1] KANKI, B.G., C.L. BRASIL. Analysis of ramp damage incidents and implications for future composite aircraft structure. NASA Technical Report, 2009.

[2] BuLL, D.J., S.M. SPEARING, I. SINCLAIR, L. HELFEN. Three-dimensional assessment of low velocity impact damage in particle toughened composite laminates using micro-focus X-ray computed tomography and synchrotron radiation laminography. In: Composites Part $A$ : Applied Science and Manufacturing, 2013, 52, 62-69. Doi:10.1016/j.compositesa.2013.05.003

[3] KRAUTKRÄMER, J., H. KRAUTKRÄMER. Werkstoffprüfung mit Ultraschall: SpringerVerlag, 2013. ISBN 3662134268.

[4] Yang, P., R. ElhajJar. Porosity content evaluation in carbon-fiber/epoxy composites using X-ray computed tomography. In: PolymerPlastics Technology and Engineering, 2014, 53(3), 217-222.

[5] Maierhofer, C., P. Myrach, M. Reischel, H. Steinfurth, M. RÖlLig, M. KUneRT. Characterizing damage in CFRP structures using flash thermography in reflection and transmission configurations. In: Composites Part B: Engineering, 2014, 57, 35-46. Doi:10.1016/j.compositesb.2013.09.036

[6] Mignogna, R.B., R.E. GReEN, J.C. Duke, E.G. HENNEKE, K.L. REIFSNIDER. Thermographic investigation of high-power ultrasonic heating in materials. In: Ultrasonics, 1981, 19(4), 159IN1161-160IN2163.

[7] Renshaw, J., S.D. Holland, R.B. ThOmpson. Measurement of crack opening stresses and crack closure stress profiles from heat generation in vibrating cracks. In: Applied Physics Letters, 2008, 93(8), 81914.

[8] Solodov, I., D. Derusova, M. Rahammer. Thermosonic Chladni figures for defectselective imaging. In: Ultrasonics, 2015, 60, 15.

[9] Solodov, I., M. Rahammer, D. Derusova, G BUSSE. Highly-efficient and noncontact vibrothermography via local defect resonance. In: Quantitative InfraRed Thermography Journal, 2015, 12(1), 98-111.

[10] Rahammer, M., M. KREUTZBruck. Fouriertransform vibrothermography with frequency sweep excitation utilizing local defect resonances. In: NDT \& E International, 2017 , 86, 83-88. Doi:10.1016/j.ndteint.2016.11.012

[11] RAHAMmER, M., M. KREUTZBRUCK. Validierung der Resonanten Frequenzsweep-Thermografie mittels einer POD-Analyse. In: Materials Testing, 2018, 60(5), 483-488.

Doi:10.3139/120.111179 